\title{
PHOLIDOTA CHINENSIS ALLEVIATES AZOXYMETHANE/DEXTRAN SULFATE SODIUM-INDUCED COLORECTAL CARCINOGENESIS THROUGH INHIBITION OF TLR4 AND COX-2
}

\section{Li-Long Liu ${ }^{1 \#}$, Kai Xu ${ }^{1 \#}$, Lu-Mei Kang ${ }^{2 \#}$, Shu-Ping Jiang ${ }^{1}$, Qing-Hua Wang ${ }^{1}$, Lei Zhang ${ }^{1}$, Shao-Gui Wan ${ }^{3}$, Hong-Ping Chen ${ }^{1^{*}}$}

\author{
${ }^{1}$ Department of Histology and Embryology, Medical College, Nanchang University, Bayi Road 461, Nanchang \\ 330006, China. \\ ${ }^{2}$ Department of Animal Science, Medical College, Nanchang University, Bayi Road 461, Nanchang 330006, China. \\ ${ }^{3}$ Institutes of Pharmacy, Pharmaceutical College of Henan University, Kaifeng, 475004, China.
}

*E-mail: jxchp2000@126.com."

Authors contributed equally to this work.

\begin{abstract}
Background: Inflammatory bowel disease (IBD) always progresses to colorectal cancer (CRC) which is the second most frequent cause of death by cancer. It is about $2 \%$ of population in the lifetime worldwide who at the risk for development of CRC. Oxaliplatin is an effective anticancer drug used for the treatment of advanced CRC; however, it always causes a robust painful neuropathy. Pholidota chinensis is a Chinese folk herbal medicine which was used for treatment of inflammation such as gastroenteritis, duodenal ulcer and bronchitis.

Materials and Methods: The azoxymethane (AOM) and dextran sulfate sodium (DSS) were used to induce the colon tumor of mice. The effect of Pholidota chinensis on colon tumorigenesis was evaluated. Immunohistochemistry and semi-quantitative RT-PCR were used to detect the expression of toll-like receptor 4 (TLR4) and cyclooxygenase-2 (COX-2) in colon.

Results: Pholidota chinensis can alleviate the colon tumorigenesis. The prevention effects of Pholidota chinensis are similar to oxaliplatin. Specifically, administration of Pholidota chinensis solution suppresses the expression of the proliferating cell nuclear antigen (PCNA), toll-like receptor 4 (TLR4) and cyclooxygenase-2 (COX-2).

Conclusion: Our findings suggested that Pholidota chinensis participate in the regulation of colon cancer development through inhibiting the expression of TLR4 and COX-2.
\end{abstract}

Key words: Pholidota chinensis; colorectal cancer; Toll-like receptor 4; Cyclooxygenase-2

\section{Introduction}

Colorectal cancer (CRC) is the third most common diagnosed cancer in males that comes after lung and prostate cancer, and the second most frequent cancer in females after breast cancer, with an estimated 1.4 million cases and 693,900 deaths occurring in 2012 (Torre et al., 2015 ). Epidemiological and experimental studies suggest that CRC is highly related to inflammatory disease (IBD) including Crohn's disease (CD) and ulcerative colitis (UC). Approximately 1.4 million Americans suffer from the IBD (Loftus and Sandborn, 2002). Patients with IBD have a significant risk for the development of colorectal cancer and the cancer risk is increased in both UC and CD (Manninen et al., 2013). Alleviating inflammation is a vital strategy for chemoprevention. The chemoprevention requires the identification of safe drugs which have less potential harm to the patients.

Numerous effective drugs, including Aspirin, non steroidal anti-inflammatory drugs (NSAIDs), celecoxib and oxaliplatin were used to treat colorectal cancer. However most of the drugs have toxic effects. Aspirin has the potential risk of inducing stroke. In terms of toxicity, the use of aspirin is associated with an increased risk in the incidence of gastrointestinal bleeding (Manzano and Perez-Segura, 2012). Meta-analyses suggested that a cardiovascular toxicity profile is presented with NSAID use, which is related to dose and duration of treatment (Kearney et al., 2006). Due to the potential toxic side-effects, NSAIDs have not been recommended to use as chemo-preventive drugs. Oxaliplatin is a 
Liu et al., Afr J Tradit Complement Altern Med. (2015) 12(6):113-121

http://dx.doi.org/10.4314/ajtcam.v12i6.11

chemotherapeutic compound widely used for treating colorectal cancer. Oxaliplatin increases apoptosis and down-regulates of NF-kB in vitro (Du Plessis-Stoman et al., 2011). However, oxaliplatin can induce various forms of nephrotoxicity such as renal tubular vacuolization, acute tubular necrosis, renal tubular acidosis, and acute kidney injury secondary to hematological toxicity (Joybari et al., 2014). Moreover, its dose-limiting toxicity is the development of a painful neuropathic syndrome sustain (Xiao and Bennett, 2012), which can lead to discontinuation of chemotherapy. Thus, it is necessary to explore the nontoxic chemicals such as herbal medicines to inhibit, retard, or reverse the process of multistage carcinogenesis.

Pholidota chinensis, "Shi-Xian-Tao" in Chinese, is a kind of traditional medicinal plant distributed mainly in the southeast of China. In Chinese folk medicine, the whole plant of Pholidota chinensis had been used to treat many kinds of disease, such as hypertension, headache, toothache, gastroenteritis, duodenal ulcer and bronchitis (Wang et al., 2006; Rueda et al., 2014). Moreover, it identifies that Stilbene derivatives from Pholidota chinensis have the potential activity of anti-inflammation in vitro (Wang et al., 2006). Thus, we wonder whether Pholidota chinensis prevents the development of colorectal cancer.

In this study, we explored the possibility that Pholidota chinensis can alleviate the colon carcinogenesis of mice which was induced by azoxymethane (AOM) plus dextran sulfate sodium (DSS). We subsequently investigated whether the Pholidota chinensis has an effect on cancer prevention we identified is mediated by cancer relevant mediators including TLR4 and COX-2.

\section{Materials and Methods}

Animal Care and Experimental Design

Female BALB/c mice weighing 21-24 g (10 weeks) were provided by the Center of Laboratory Animal Science of Nanchang University. The mice were fed a standard laboratory diet under controlled temperature and 12 -h light/dark cycle at $20-22^{\circ} \mathrm{C}$. The animals had free access to dietary food and water except during the test sessions. All experimental procedures were approved by the Institutional Animal Care and Use Committee of the Medical College of Nanchang University. All efforts were made to minimize animal suffering and to reduce the number of animals used. Mice were randomly divided into 3 groups with 12 mice in each group: a control group, an oxaliplatin treatment group, and a Pholidota chinensis treatment group (Figure 1).

\section{AOM/DSS-Induced Colitis-Associated Carcinogenesis}

The mice were injected intraperitoneally with a single dose of $12 \mathrm{mg} / \mathrm{kg}$ body weight of azoxymethane (AOM) (Sigma-Aldrich, USA) dissolved in $0.9 \%$ saline. The day was recorded as day 0. Five days after AOM administration, 2.5\% DSS (MP Biomedicals, USA; MW, 36-50 $\mathrm{kDa}$ ) was administered through the sterile deionized water for 5 consecutive days, followed by 10 days of drinking water. This DSS cycle was again repeated twice, and then regular water was administered until the end of the experiment at day 100 (Figure 1).

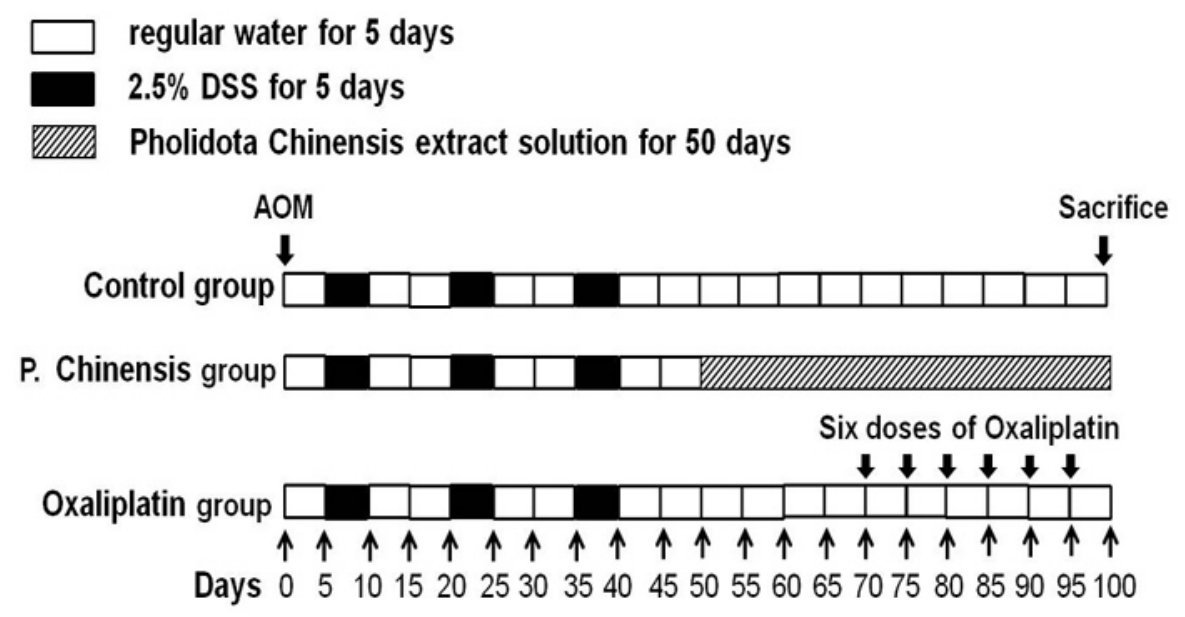

Figure1: Experimental protocol. Mice were divided into three groups, i.e., control (AOM/DSS control; n=12) group, P. Chinensis (Pholidota chinensis; $\mathrm{n}=12)$ group and oxaliplatin $(\mathrm{n}=12)$ group. All mice received a single intraperitoneal injection of azoxymethane $(\mathrm{AOM} ; 12 \mathrm{mg} / \mathrm{kg})$ at 
Liu et al., Afr J Tradit Complement Altern Med. (2015) 12(6):113-121

http://dx.doi.org/10.4314/ajtcam.v12i6.11

day 0. Five days after the AOM administration, mice received 2.5\% dextran sodium sulfate (DSS) in drinking water for 5 consecutive days, followed by 10 days of drinking water. This DSS cycle was again repeated twice. Mice in the Pholidota chinensis group also received Pholidota chinensis extract solution in a concentration of thirty grams of pharmaceutical medicine in one liter of drinking water since days of 50 for 50 consecutive days. Mice in the oxaliplatin group also received 6 doses of oxaliplatin (intraperitoneal injection, $10 \mathrm{mg} / \mathrm{kg}$ ) since days of 70 once every 5 days for 30 days.

\section{Preparation of Pholidota Chinensis Extract}

The powdered and shade-dried whole plants of Pholidota chinensis (Chinese Medicine Store; $1.0 \mathrm{~kg}$ ) were stewed twice with deionized water $(10 \mathrm{~L})$ for 2.0 hours. The deionized water extract were combined and filtered with medicine gauze. The solution was added $95 \%$ ethanol to the concentration of $60 \%$, mixed thoroughly and kept static for 48 hours. Then, the $60 \%$ ethanol extract solution was concentrated to two grams of pharmaceutical medicine in one milliliter of distilled water with Rotating Evaporation Instrument. The solution was stored at $4^{\circ} \mathrm{C}$ for using.

\section{Drug Administration}

In the oxaliplatin treatment group (oxaliplatin group), the mice were injected intraperitoneally with a dose of $10 \mathrm{mg} / \mathrm{kg}$ body weight of oxaliplatin (Qilu Pharmaceutical Company, China) dissolved in 5\% glucose solution. The drugs were delivered once every 5 days for 30 days, beginning on day 70. A total of six doses of oxaliplatin were delivered. In the Pholidota chinensis treatment group (P. Chinensis group), the regular water was changed to the Pholidota chinensis extract solution in a concentration of thirty grams of pharmaceutical medicine in one liter of drinking water since days of 50 for 50 consecutive days. At day 100, all the mice were deeply anesthetized with pentobarbital sodium (100 $\mathrm{mg} / \mathrm{kg}$ sodium pentobarbital, i.p.) and sacrificed by decapitation.

\section{Histopathological Examination}

Entire colons were excised, cut longitudinally, rinsed with ice-cold PBS for 3 times. Tumor numbers were counted and recorded under the anatomy microscope. The tumors were classified based on the diameter.

\section{Immunohistochemistry}

The Formalin-fixed, paraffin-embedded tissues were cut, and sections ( $5 \mu \mathrm{m}$ thickness) were used for Immunohistochemistry (IHC) detection. IHC was carried out as previously described (Li et al., 2013), using primary antibodies against TLR4 (Boster, China), COX-2 (Abcam, USA) and proliferating cell nuclear antigen (PCNA; A0264, ABclonal, USA). The rabbit kit (PV-6001, ZSGB-BIO, China) was used as secondary antibody according to the instruction. Protein localization was detected following incubation with diaminobenzidine (DAB; Sigma, USA) and $\mathrm{H}_{2} \mathrm{O}_{2}$ for 2 min. Finally, sections were dehydrated in graded alcohols and mounted with neutral balsam.

\section{Semi-quantitative RT-PCR}

Total RNA from mice colon was isolated using TRIzol (Invitrogen, Carlsbad, CA, USA) according to the manufacturer's protocol. 1000 ng total RNA was used as a template for reverse transcription using the Applied Bio-systems Reverse Transcription Kit (Applied Bio-systems, Foster City, CA, USA). Antisense primer 5'-ATA ACC TAC CTT TTC GGA GC-3' and sense primer 5'-GCT TTC ACC TCT GCC TTC AC-3' specific for TLR4. Antisense primer 5'-GAA CCC AGG TCC TCG CTT-3' and sense primer 5'-GGG AAG CCT TCT CCA ACC -3' specific for COX-2. The PCR products were amplified using the following cycling parameters: $94^{\circ} \mathrm{C}$ for $5 \mathrm{~min}$, followed by 32 cycles of $94^{\circ} \mathrm{C}$ for $45 \mathrm{~s}, 55^{\circ} \mathrm{C}$ for $40 \mathrm{~s}$, and $72^{\circ} \mathrm{C}$ for $50 \mathrm{~s}$, and finally a single cycle at $72^{\circ} \mathrm{C}$ for 5 minutes. $\beta$-actin was used as an internal positive control. 
Liu et al., Afr J Tradit Complement Altern Med. (2015) 12(6):113-121

http://dx.doi.org/10.4314/ajtcam.v12i6.11

Statistical Analysis

Data indicate the mean \pm S.D. Comparisons of means between two groups were done with test and those between multiple groups with one way analysis of variance (ANOVA). A value of $P<0.05$ was considered statistically significant.

\section{Results}

Pholidota Chinensis Administration Alleviates the Body Weight Loss Induced by AOM/DSS

The bodyweight of the mice were evaluated (Figure 2). At the first day of AOM injection (day 0), the body weight of the mice among the three groups have no difference. At days 100 after treated by AOM, the body weight of mice in the Pholidota chinensis group were significantly higher than in the AOM/DSS control group $(P<0.01)$. However, the bodyweight of mice treated with oxipalitin were decreased when compared to both the AOM/DSS control group $(P<0.001)$ and the Pholidota chinensis group $(P<0.001)$. The results showed that Pholidota chinensis prevent the body weight loss induced by AOM/DSS. However, oxaliplatin enhanced the body weight loss caused by AOM/DSS. In the control group, the body weight of mice had no significant difference between $\mathrm{d} 0$ and d100. It suggested that AOM/DSS administration can prevent the mice growth. Thus, Pholidota chinensis attenuated the AOM/DSS-induced growth inhibition.

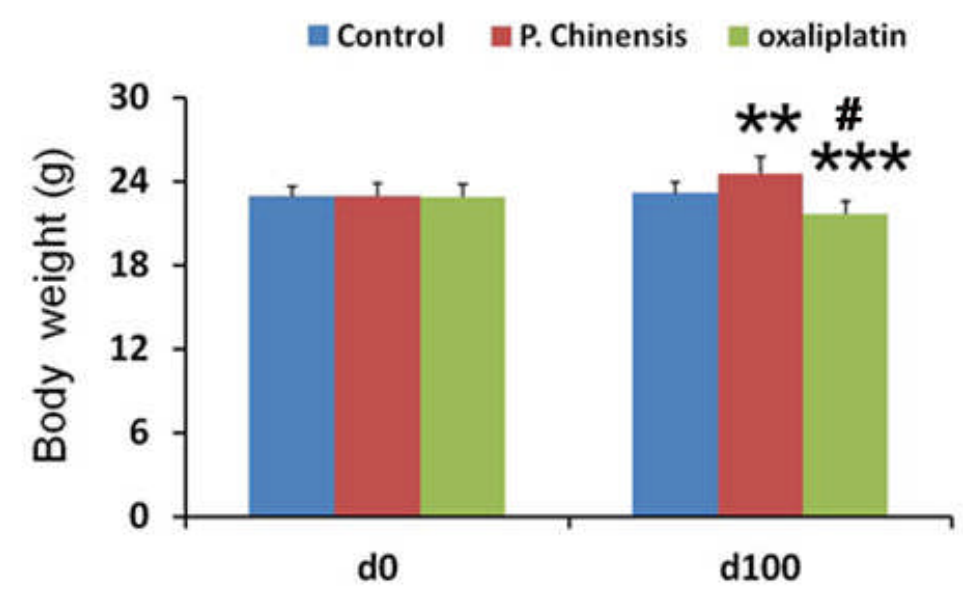

Figure 2: Body weight change induced by AOM/DSS treatment. All mice were weighed at day 0 before AOM injection. After the mice sacrificed at days 100 , all the mice were weighed. Data is expressed and represented as mean $\pm \mathrm{S} . \mathrm{D}, \mathrm{n}=12$. $^{* *} P<0.01$ and ${ }^{* * *} P<0.01 v s$ control group, ${ }^{\#} P<0.01$ vs Pholidota chinensis group.

\section{Pholidota Chinensis Administration Attenuates Susceptibility towards AOM/DSS Induced Colonic Carcinogenesis}

At day 100 after treated by AOM, the tumors were observed and calculated under the anatomy microscope (Figure 3). The tumor numbers based on the diameter were calculated. Both Pholidota chinensis $(P<0.01)$ and oxaliplatin $(P<0.001)$ inhibited the development of tumor whose diameter is between $1 \mathrm{~mm}$ and $3 \mathrm{~mm}$. However, Pholidota chinensis and oxaliplatin had no influence on the development of tumor whose diameter is less than $1 \mathrm{~mm}$ and over $3 \mathrm{~mm}$, respectively (Figure 3B). In addition, the total number of tumors in both Pholidota chinensis group ( $P$ $<0.01)$ and oxaliplatin group $(P<0.001)$ were significantly reduced when compared to the AOM/DSS control group (Figure 3C). The results indicated that Pholidota chinensis can inhibit the development of colorectal tumor induced by AOM/DSS. The anti-tumor role of Pholidota chinensis is similar to oxaliplatin. 
Liu et al., Afr J Tradit Complement Altern Med. (2015) 12(6):113-121

http://dx.doi.org/10.4314/ajtcam.v12i6.11

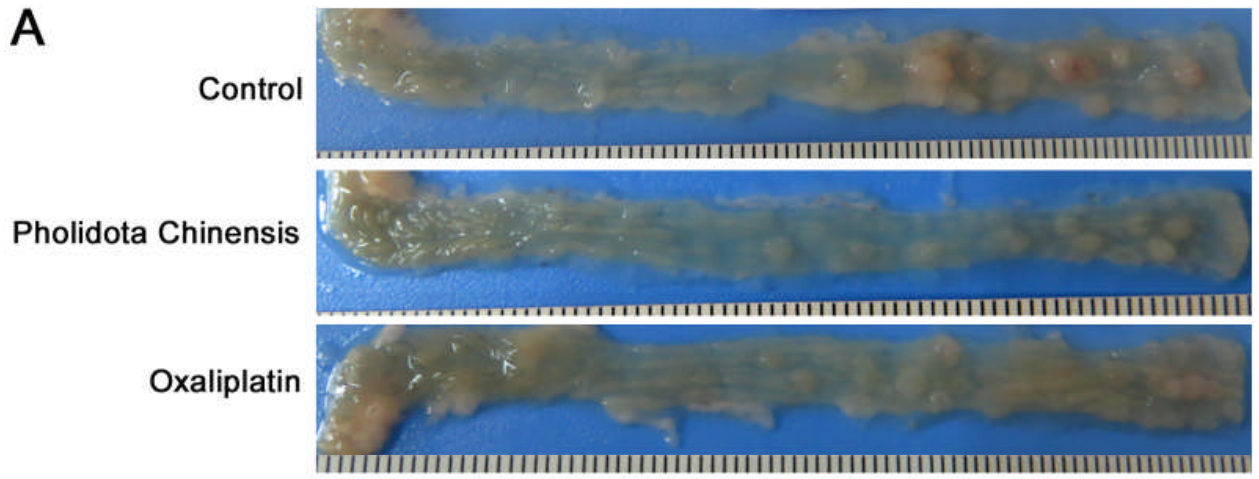

B

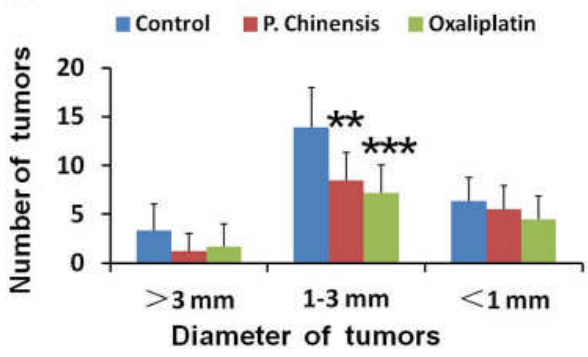

C

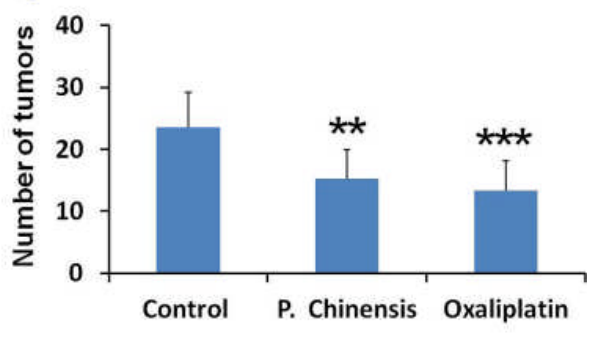

Figure 3: Tumorigenesis induced by AOM/DSS treatment. At day 100, all mice were sacrificed and the tumor multiplicity was evaluated. (A) Representative pictures of tumor in colon. (B) Average tumor numbers in each colon based on the tumor diameters. (C) Average total tumor numbers in each colon. Data is expressed and represented as mean $\pm \mathrm{S} . \mathrm{D}, \mathrm{n}=12 .{ }^{* *} P<0.01$ and ${ }^{* * *} P<0.01$ vs control group.

Pholidota chinensis administration decreases TLR4 and COX-2 mRNA expression in the colon of mice treated with AOM/DSS

To determine the functional role of Pholidota chinensis on tumor prevention at a molecular level, we examined whether Pholidota chinensis administration would modulate the mRNA expression of TLR4 and COX-2. The results suggested that Pholidota chinensis inhibits both TLR4 $(P<0.05)$ and COX-2 $(P<0.01)$ mRNA expression in the colon of mice which treated with AOM/DSS (Figure 4).

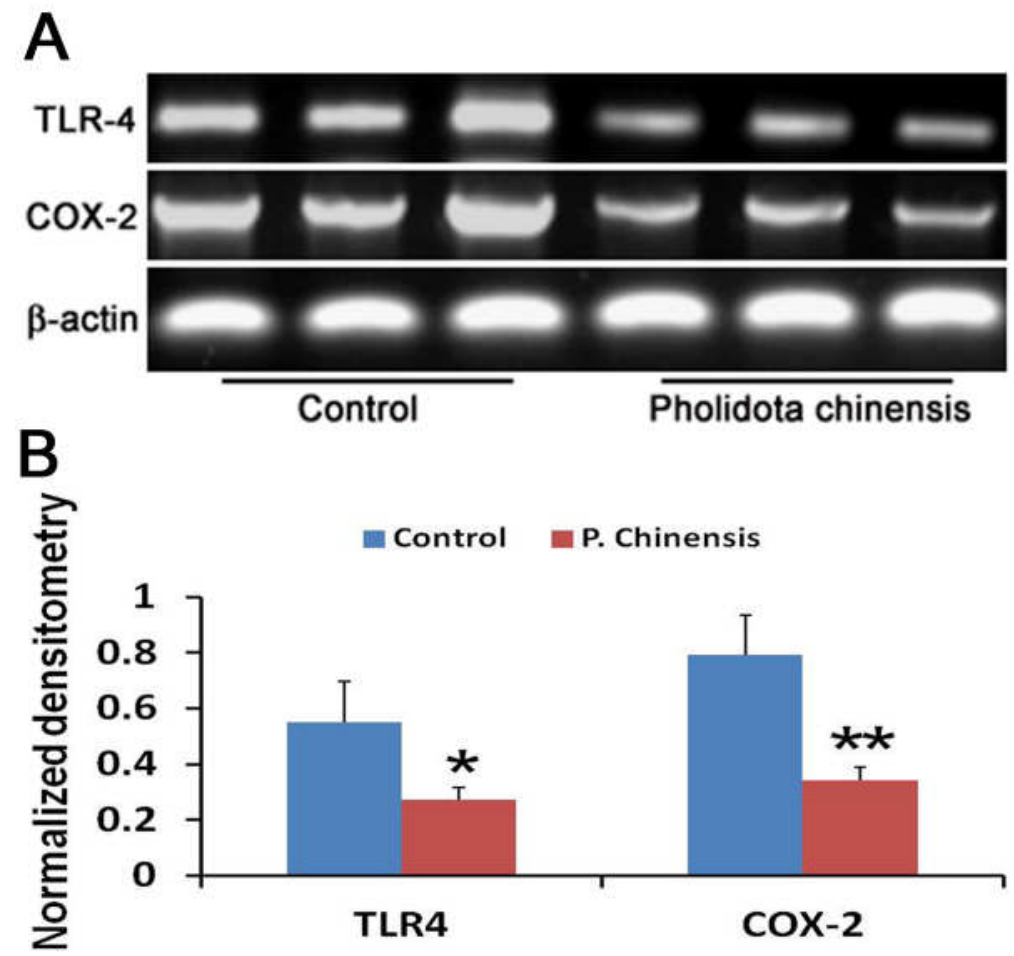

Figure 4: Pholidota chinensis suppressed the mRNA levels of TLR4 and COX-2. (A) TLR4 and COX-2 mRNA levels in colon were detected by Semi-quantitative RT-PCR. (B) Quantitative data indicating expressions of the mRNA levesl. Data is expressed and represented as mean \pm S.D., 
Liu et al., Afr J Tradit Complement Altern Med. (2015) 12(6):113-121

http://dx.doi.org/10.4314/ajtcam.v12i6.11

$\mathrm{n}=3$. $^{*} P<0.05 v s$ control group and ${ }^{* *} P<0.01$ vs control group.

Pholidota chinensis administration reduces immunoreactivities of TLR4, COX-2 and PCNA in the colon of mice treated with AOM/DSS

The immunoreactivities of TLR4, COX-2 and PCNA were detected by immunohistochemistry (Figure 5). The percentage of the positive cells of TLR4, COX-2 and PCNA was analysed. The results showed that Pholidota chinensis administration reduces the immunoreactivities of TLR4 $(P<0.01)$, COX-2 $(P<0.001)$ and PCNA $(P<0.01)$.

A

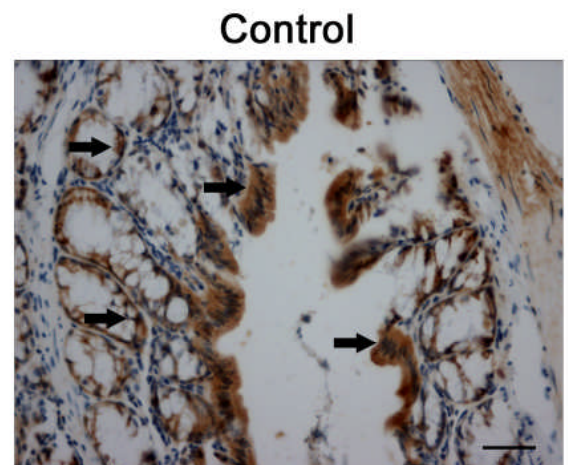

Pholidota chinensis

TLR4

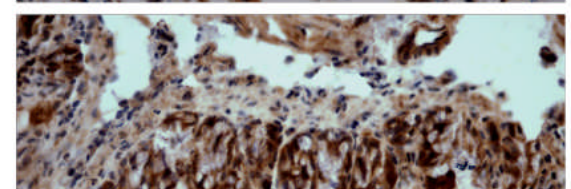

coX-2
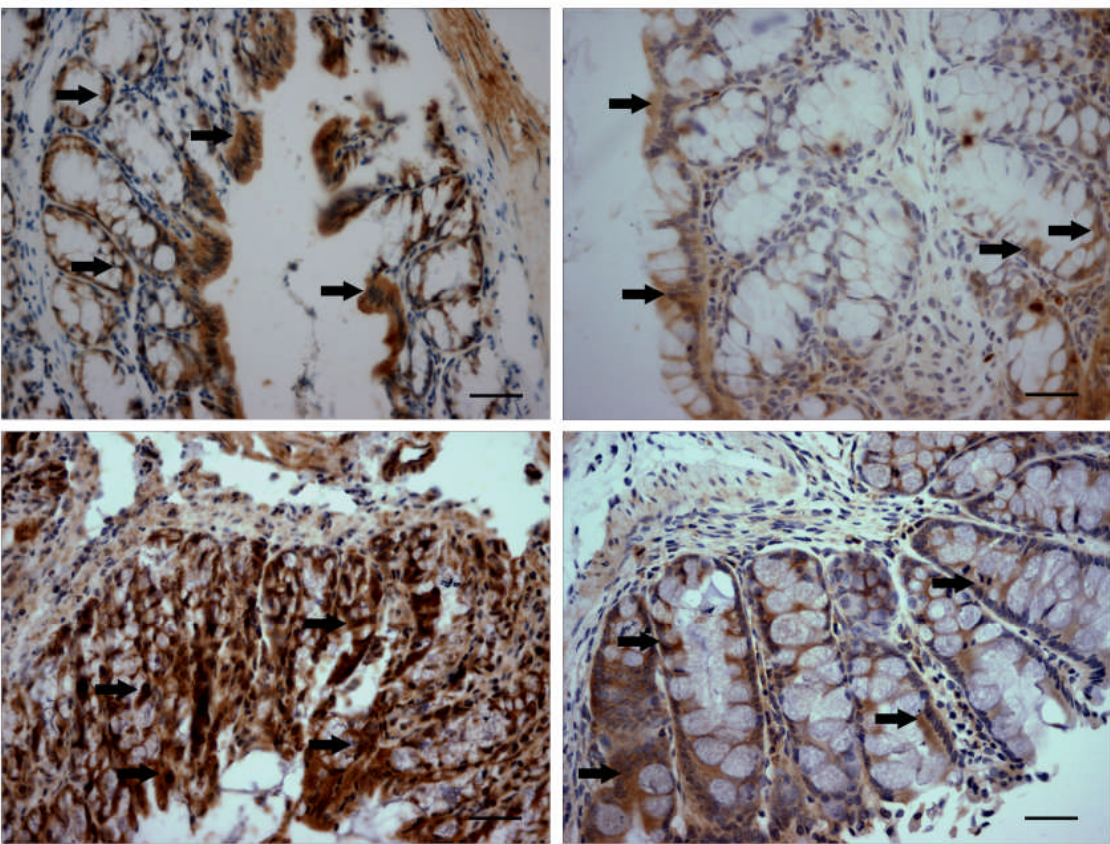

PCNA
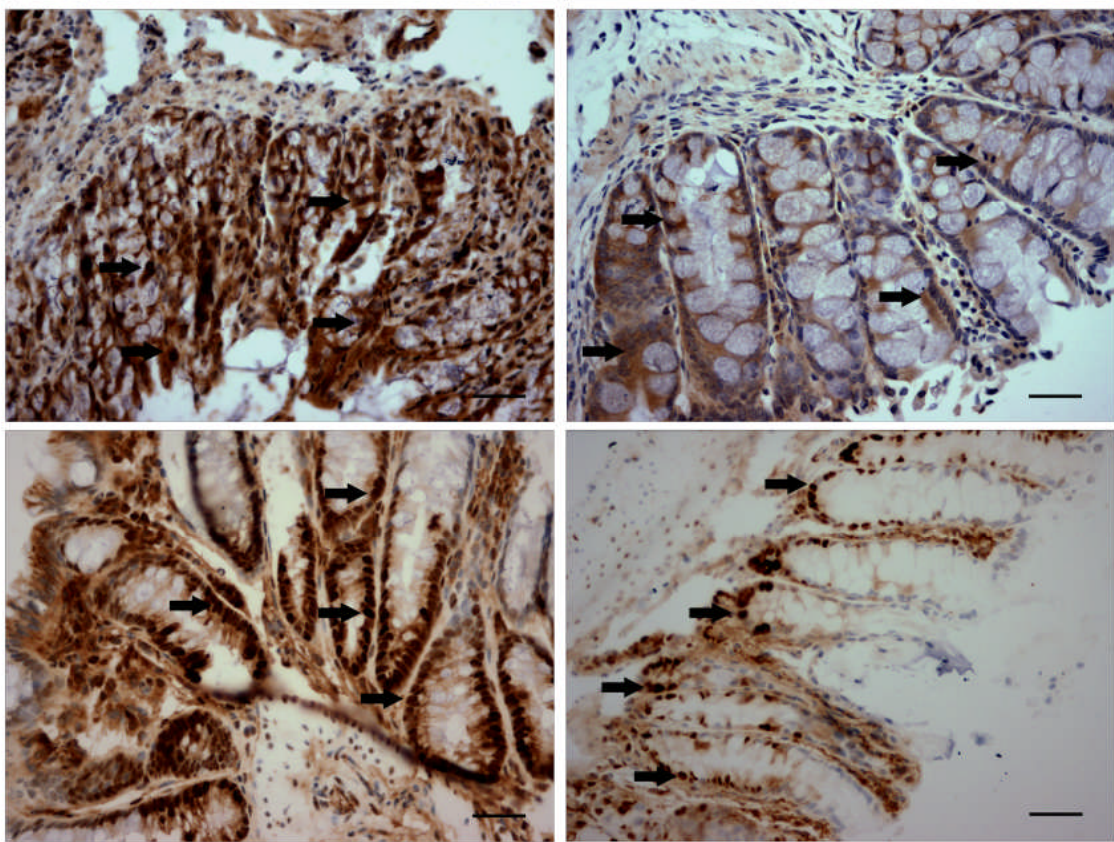

B

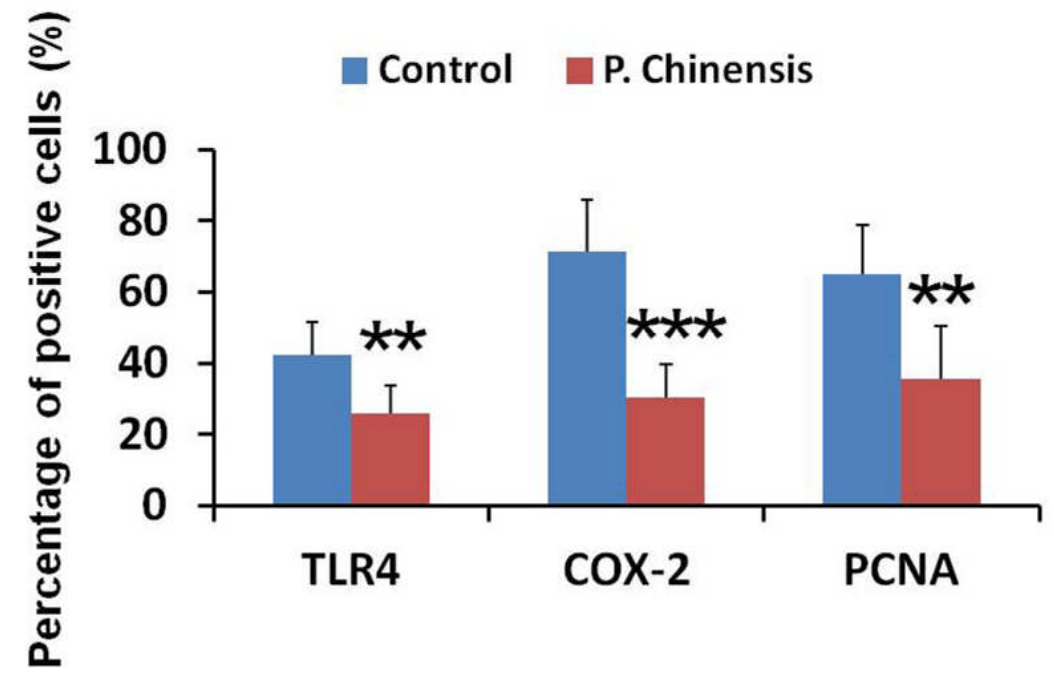




\section{Liu et al., Afr J Tradit Complement Altern Med. (2015) 12(6):113-121}

\section{http://dx.doi.org/10.4314/ajtcam.v12i6.11}

Figure 5: Pholidota chinensis decreased the immunoreactivities of TLR4, COX-2 and PCNA in colon of mice treated by AOM/DSS. (A) The immunereactivities of TLR4, COX-2 and PCNA in colon of mice of control and Pholidota chinensis groups were detected by immunohistochemisty. In each group, colons from six mice were detected. In each colon, five paraffin slides were used. In each slide, five high-powered fields (HPF, 40x) were selected. (B) The percentages of the positive cells of TLR4, COX-2 and PCNA on HPF were analysed. Scale bar is $40 \mu \mathrm{m}$. Arrow indicate the typical immuno-positive cells. Data is expressed and represented as mean \pm S.D. $n=6$. $^{* *} P<0.01$ and ${ }^{* * *} P$ $<0.001$ vs control group.

\section{Discussion}

Cancer is a global health problem with severe morbidity and mortality, although numerous treatments of chemoprevention and chemotherapy have been developed. As we all know, treatment of cancer with chemical reagents always cause serious side-effects. Especially, unavoidable long-term treatment-related side-effects used to make the cancer survivors face defeat. Thus, exploring nontoxic herbal medicines to attenuate the carcinogenesis is needed. We showed for the first time that Pholidota chinensis extract alleviated the colonic tumor progression. The prevention of colon cancer may be mediated by inhibiting expressions of TLR4 and COX-2.

Toll-like receptors (TLRs) activate both the innate immune system and the adaptive immune system in response to cancer. Different TLR ligands play variety of roles in various tumor developments. TLR3 can induce apoptosis of human breast and prostate cancer cells and inhibit tumor growth in vivo (Okamoto and Sato, 2003; Salaun et al., 2006; Gambara et al., 2015). By contrast, TLR4-mediated signaling has been identified in tumor cell invasion, survival, and metastasis in a variety of cancers (Yang et al., 2014; Wang et al., 2014; Hua et al., 2009; Szczepanski et al., 2009). Moreover, numerous investigations imply that TLR4 mediates the colorectal cancer process (Tchorzewski et al., 2014; Santaolalla et al., 2013; Fukata et al., 2011). Activation of TLR-4 signaling accelerates colon cancer cell adhesion via NF-kappaB mediated transcriptional up-regulation of Nox-1 (O'Leary et al., 2012). Furthermore, Inhibition of TLR4 attenuates up-regulation of NF- $\kappa B$ and inhibits colon tumor growth (Sun et al., 2008). These findings suggest that targeting TLR4 signaling may be a potential mechanism to abrogate the progression of colorectal tumor which mediated by inflammation.

It is well known that patients with inflammatory bowel disease face higher risk of CRC. Inflammation involves interaction between various immune cells, inflammatory cells, chemokines, cytokines, and pro-inflammatory mediators, such as cyclooxygenase-2 (COX-2). COX-2 and its enzymatic product prostaglandin E2 (PGE2) have key roles in promoting the development of CRC (Castellone et al., 2006). Over-expression of COX-2 promoted azoxymethane (AOM) induced tumor progression by activating EGFR and Akt signaling pathways (Al-Salihi et al., 2009). All primary carcinomas of the colon and rectum (100\%) and 56 out of the 57 liver metastases $(98.2 \%)$ showed different degrees of cytoplasmatic COX-2 expression. And COX-2 inhibition can contribute to the anticancer roles of CRC through anti-apoptotic effects and anti-angiogenic actions (Kasper et al., 2010). The use of COX-2 inhibitor may be associated with improved outcomes in stage III colon cancer patients (Ng et al., 2015). Thus, target COX-2 is a potential strategy for treatment of CRC.

It is identified that the component of Pholidota chinensis, such as Stilbene, has the role of anti-inflammation (Wang et al., 2006). This implies that Pholidota chinensis may attenuate the cancer progression by inhibition of inflammation. Recently, it has showed that phoyunbene B, extracted from Pholidota yunnanensis, can induce G2/M cell cycle arrest and apoptosis in HepG2 liver cancer cells. In current research, we found that Pholidota chinensis extract solution can reduce the expressions of TLR4, receptor of inflammation mediators s100a8/s100a9 and COX-2, a pro-inflammatory mediators in the colon of mice which treated by AOM/DSS.

\section{Conclusions}

In conclusion, the present study has firstly shown that Pholidota chinensis solution can attenuate the CRC progression induced by AOM/DSS. In addition, Pholidota chinensis solution reduced the expressions of both TLR4 and COX-2 in the colon of CRC mice. These data illustrated that the CRC prevention of Pholidota chinensis might be mediated by inhibition of TLR4 and COX-2. However, the active ingredients of Pholidota chinensis of CRC prevention need to be further investigated. We hope that our findings will promote the design more effectively and specific drugs with fewer side-effect for treatment of the human CRC. 


\section{Liu et al., Afr J Tradit Complement Altern Med. (2015) 12(6):113-121 \\ http://dx.doi.org/10.4314/ajtcam.v12i6.11 \\ Acknowledgment}

This study was supported by the National Natural Science Foundation of China (Nos. 81260318 and 81201578 ); Educational Department Foundation of Jiangxi Province (GJJ14162); Foundation of Health and Family Planning Commission of Jiangxi Providence (20143198); National Innovation Experiment Program for University Students (20140403050) and Provincial Innovation Experiment Program for University Students (201310403032).

\section{References}

1. Al-Salihi, M. A., Terrece Pearman, A., Doan, T., Reichert, E. C., Rosenberg, D. W., Prescott, S. M., Stafforini, D. M. and Topham, M. K. (2009). Transgenic expression of cyclooxygenase-2 in mouse intestine epithelium is insufficient to initiate tumorigenesis but promotes tumor progression. Cancer letters, 273: 225-232.

2. Castellone, M. D., Teramoto, H. and Gutkind, J. S. (2006). Cyclooxygenase-2 and colorectal cancer chemoprevention: the beta-catenin connection. Cancer research, 66: 11085-11088.

3. Du Plessis-Stoman, D., du Preez, J. and van de Venter, M. (2011). Combination treatment with oxaliplatin and mangiferin causes increased apoptosis and downregulation of NFKB in cancer cell lines. African Journal of Traditional Complementary and Alternative Medicines, 8: 177-184.

4. Fukata, M., Shang, L., Santaolalla, R., Sotolongo, J., Pastorini, C., Espana, C., Ungaro, R., Harpaz, N., Cooper, H. S., Elson, G., Kosco-Vilbois, M., Zaias, J., Perez, M. T., Mayer, L., Vamadevan, A. S., Lira, S. A. and Abreu, M. T. (2011). Constitutive activation of epithelial TLR4 augments inflammatory responses to mucosal injury and drives colitis-associated tumorigenesis. Inflammatory bowel diseases, 17: 1464-1473.

5. Gambara, G., Desideri, M., Stoppacciaro, A., Padula, F., De Cesaris, P., Starace, D., Tubaro, A., Del Bufalo, D., Filippini, A., Ziparo, E. and Riccioli, A. (2015). TLR3 engagement induces IRF-3-dependent apoptosis in androgen-sensitive prostate cancer cells and inhibits tumour growth in vivo. Journal of cellular and molecular medicine, 19: 327-339.

6. Hua, D., Liu, M. Y., Cheng, Z. D., Qin, X. J., Zhang, H. M., Chen, Y., Qin, G. J., Liang, G., Li, J. N., Han, X. F. and Liu, D. X. (2009). Small interfering RNA-directed targeting of Toll-like receptor 4 inhibits human prostate cancer cell invasion, survival, and tumorigenicity. Molecular immunology, 46: 2876-2884.

7. Joybari, A. Y., Sarbaz, S., Azadeh, P., Mirafsharieh, S. A., Rahbari, A., Farasatinasab, M. and Mokhtari, M. (2014). Oxaliplatin-induced renal tubular vacuolization. The Annals of pharmacotherapy, 48: 796-800.

8. Kasper, H. U., Konze, E., Dienes, H. P., Stippel, D. L., Schirmacher, P. and Kern, M. (2010). COX-2 expression and effects of COX-2 inhibition in colorectal carcinomas and their liver metastases. Anticancer research, 30: 2017-2023.

9. Kearney, P. M., Baigent, C., Godwin, J., Halls, H., Emberson, J. R. and Patrono, C. (2006). Do selective cyclo-oxygenase-2 inhibitors and traditional non-steroidal anti-inflammatory drugs increase the risk of atherothrombosis? Meta-analysis of randomised trials. Bmj, 332: $1302-1308$.

10. Li, X., Kang, L., Li, G., Zeng, H., Zhang, L., Ling, X., Dong, H., Liang, S. and Chen, H. (2013). Intrathecal leptin inhibits expression of the P2X2/3 receptors and alleviates neuropathic pain induced by chronic constriction sciatic nerve injury. Molecular pain, 9: 65 .

11. Loftus, E. V., Jr. and Sandborn, W. J. (2002). Epidemiology of inflammatory bowel disease. Gastroenterology clinics of North America, 31: $1-20$.

12. Manninen, P., Karvonen, A. L., Huhtala, H., Aitola, P., Hyoty, M., Nieminen, I., Hemminki, H. and Collin, P. (2013). The risk of colorectal cancer in patients with inflammatory bowel diseases in Finland: a follow-up of 20 years. Journal of Crohn's \& colitis, 7: e551-557.

13. Manzano, A. and Perez-Segura, P. (2012). Colorectal cancer chemoprevention: is this the future of colorectal cancer prevention? The Scientific World Journal, 2012: 327341.

14. Ng, K., Meyerhardt, J. A., Chan, A. T., Sato, K., Chan, J. A., Niedzwiecki, D., Saltz, L. B., Mayer, R. J., Benson, A. B., 3rd, Schaefer, P. L., Whittom, R., Hantel, A., Goldberg, R. M., Venook, A. P., Ogino, S., Giovannucci, E. L. and Fuchs, C. S. (2015). Aspirin and COX-2 Inhibitor Use in Patients With Stage III Colon Cancer. Journal of the National Cancer Institute, 107: 345. 


\section{Liu et al., Afr J Tradit Complement Altern Med. (2015) 12(6):113-121}

\section{http://dx.doi.org/10.4314/ajtcam.v12i6.11}

15. O'Leary, D. P., Bhatt, L., Woolley, J. F., Gough, D. R., Wang, J. H., Cotter, T. G. and Redmond, H. P. (2012). TLR-4 signalling accelerates colon cancer cell adhesion via NF-kappaB mediated transcriptional up-regulation of Nox-1. PloS one, 7: e44176.

16. Okamoto, M. and Sato, M. (2003). Toll-like receptor signaling in anti-cancer immunity. The journal of medical investigation, 50: 9-24.

17. Rueda, D. C., Schoffmann, A., De Mieri, M., Raith, M., Jahne, E. A., Hering, S. and Hamburger, M. (2014). Identification of dihydrostilbenes in Pholidota chinensis as a new scaffold for GABAA receptor modulators. Bioorganic \& medicinal chemistry, 22: 1276-1284.

18. Salaun, B., Coste, I., Rissoan, M. C., Lebecque, S. J. and Renno, T. (2006). TLR3 can directly trigger apoptosis in human cancer cells. Journal of immunology, 176: 4894-4901.

19. Santaolalla, R., Sussman, D. A., Ruiz, J. R., Davies, J. M., Pastorini, C., Espana, C. L., Sotolongo, J., Burlingame, O., Bejarano, P. A., Philip, S., Ahmed, M. M., Ko, J., Dirisina, R., Barrett, T. A., Shang, L., Lira, S. A., Fukata, M. and Abreu, M. T. (2013). TLR4 activates the beta-catenin pathway to cause intestinal neoplasia. PloS one, 8: e63298.

20. Sun, Q., Liu, Q., Zheng, Y. and Cao, X. (2008). Rapamycin suppresses TLR4-triggered IL-6 and PGE(2) production of colon cancer cells by inhibiting TLR4 expression and NF-kappaB activation. Molecular immunology, 45: 2929-2936.

21. Szczepanski, M. J., Czystowska, M., Szajnik, M., Harasymczuk, M., Boyiadzis, M., Kruk-Zagajewska, A., Szyfter, W., Zeromski, J. and Whiteside, T. L. (2009). Triggering of Toll-like receptor 4 expressed on human head and neck squamous cell carcinoma promotes tumor development and protects the tumor from immune attack. Cancer research, 69: 3105-3113.

22. Tchorzewski, M., Lewkowicz, P., Dziki, A. and Tchorzewski, H. (2014). Expression of toll-like receptors on human rectal adenocarcinoma cells. Archivum immunologiae et therapiae experimentalis, 62: 247-251.

23. Torre, L. A., Bray, F., Siegel, R. L., Ferlay, J., Lortet-Tieulent, J. and Jemal, A. (2015). Global cancer statistics, 2012. Global cancer statistics, 2012. CA: a cancer journal for clinicians. 65: 87-108.

24. Wang, A. C., Ma, Y. B., Wu, F. X., Ma, Z. F., Liu, N. F., Gao, R., Gao, Y. S. and Sheng, X. G. (2014). TLR4 induces tumor growth and inhibits paclitaxel activity in MyD88-positive human ovarian carcinoma. Oncology letters, 7: 871-877.

25. Wang, J., Matsuzaki, K. and Kitanaka, S. (2006). Stilbene derivatives from Pholidota chinensis and their anti-inflammatory activity. Chemical \& pharmaceutical bulletin, 54: 1216-1218.

26. Xiao, W. H. and Bennett, G. J. (2012). Effects of mitochondrial poisons on the neuropathic pain produced by the chemotherapeutic agents, paclitaxel and oxaliplatin. Pain, 153: 704-709.

27. Yang, H., Wang, B., Wang, T., Xu, L., He, C., Wen, H., Yan, J., Su, H. and Zhu, X. (2014). Toll-like receptor 4 prompts human breast cancer cells invasiveness via lipopolysaccharide stimulation and is overexpressed in patients with lymph node metastasis. PloS one, 9: e109980. 